\title{
A thin target approach for portal imaging in medical accelerators
}

\author{
A Tsechanski $\nmid \ddagger \|$, A F Bielajew $\S$, S Faermann $\ddagger$ and Y Krutman $\dagger \ddagger$ \\ $\dagger$ Ben-Gurion University of the Negev, Nuclear Engineering Department, Be'er Sheva, Israel \\ $\ddagger$ Ben-Gurion University of the Negev, Soroka Medical Center, Institute of Oncology, Be'er \\ Sheva, Israel \\ $\S$ The University of Michigan, Department of Nuclear Engineering and Radiological Sciences, \\ Ann Arbor, Michigan, USA
}

Received 12 December 1997

\begin{abstract}
A new thin-target method (patent pending) is described for portal imaging with low-energy (tens of $\mathrm{keV}$ ) photons from a medical linear accelerator operating in a special mode. Low-energy photons are usually produced in the accelerator target, but are absorbed by the target and flattening filter, both made of medium- or high- $Z$ materials such as $\mathrm{Cu}$ or W. Since the main contributor to absorption of the low-energy photons is self-absorption by the thick target through the photoelectric effect, it is proposed to lower the thickness of the portal imaging target to the minimum required to get the maximum low-energy photon fluence on the exit side of the target, and to lower the atomic number of the target so that predominantly photoelectric absorption is reduced. To determine the minimum thickness of the target, EGS4 Monte Carlo calculations were performed. As a result of these calculations, it was concluded that the maximum photon fluence for a $4 \mathrm{MeV}$ electron beam is obtained with a $1.5 \mathrm{~mm} \mathrm{Cu}$ target. This value is approximately five times less than the thickness of the $\mathrm{Cu}$ target routinely used for bremsstrahlung production in radiotherapeutic practice. Two sets of experiments were performed: the first with a $1.5 \mathrm{~mm}$ $\mathrm{Cu}$ target and the second with a $5 \mathrm{~mm} \mathrm{Al}$ target ( $\mathrm{Cu}$ mass equivalent) installed in the linear accelerator. Portal films were taken with a Rando anthropomorphic phantom. To emphasize the low-energy response of the new thin target we used a Kodak Min-R mammographic film and cassette combination, with a strong low-energy response. Because of its high sensitivity, only $1 \mathrm{cGy}$ is required. The new portal images show a remarkable improvement in sharpness and contrast in anatomical detail compared with existing ones. It is also shown that further lowering of the target's atomic number (for example to $\mathrm{C}$ or $\mathrm{Be}$ ) produces no significant improvement.
\end{abstract}

\section{Introduction}

A portal image is defined as any patient image taken with the treatment beam to check the accuracy of the planning target volume. It is an essential tool for accurate and reproducible routine external beam radiotherapy. However, in the $\mathrm{MeV}$ photon energy range, the mass attenuation coefficients for bone and soft tissue are nearly the same since the dominant primary attenuation mechanism is the Compton interaction, the magnitude of which is linearly dependent on the atomic number $Z$. It follows, therefore, that portal radiographs taken in the $\mathrm{MeV}$ energy range will be sensitive only to differences in density and not to differences in atomic number (on a mass for mass basis). As a result, the portal images taken in the $\mathrm{MeV}$ photon energy range suffer inherently from very low contrast and sharpness.

|| Address for correspondance: Ben-Gurion University of the Negev, Nuclear Engineering Department, PO Box 653, Be’er Sheva 84105, Israel. E-mail address: alex@ prism.bgu.ac.il 
Several techniques to improve the portal imaging quality have been suggested, for example reducing the energy of the $\mathrm{x}$-ray spectrum and/or emphasizing the response of the detector to the low-energy part of the spectrum (Karzmark et al 1993).

Improvement in the portal imaging contrast with decreasing photon energy follows directly from the decrease in the Compton cross section approximately with the two-thirds power of the photon energy (Evans 1955). The second factor in the contrast improvement with decreasing photon energy is that at higher incident photon energies, Compton scattering is mainly forward-peaked, increasing the probability that a scattered photon will still be in the field at the cassette.

In this context Amols et al (1986) compared measured and calculated film contrast on a specially designed test phantom for x-ray energies common in radiotherapy. Taking $6 \mathrm{MV}$ as a basis for comparison and a phantom-to-film distance of $30 \mathrm{~cm}$, the relative contrast values measured were 1.20 at $4 \mathrm{MV}, 1.00$ at $6 \mathrm{MV}, 0.71$ at $10 \mathrm{MV}$ and 0.60 at $15 \mathrm{MV}$. These results are in good agreement with predictions of theoretical calculations of Compton scattering. It should be pointed out that for high-energy photons $(\sim 0.5-15 \mathrm{MeV})$, Compton scatter is the dominant interaction mechanism, especially in tissue-like materials, accounting for up to $99 \%$ of the total cross section (Johns and Cunningham 1983).

Purdy et al (1981) achieved significant improvement in portal films for a Varian Clinac 20 linear accelerator (18 MV photon beam) by developing a separate portal film mode which utilizes the $6 \mathrm{MeV}$ electron beam and $18 \mathrm{MV}$ x-ray target to produce an unflattened $6 \mathrm{MV}$ $\mathrm{x}$-ray beam. Following this work Varian Associates developed an optional accessory called the 'Portal Film Option', which is based on a 6 MV unflattened beam for the Clinac series high-energy models (Varian 1982). Further improvement of the Portal Film Option in a Clinac 18 has been reported by Tsechanski et al (1997a). This was done by lowering the nominal electron beam energy in the portal film option to $4 \mathrm{MeV}$, thus increasing the relative contribution of the low-energy portion of the photon spectrum. For the same purpose, between the two existing copper target ports of different thicknesses, the thinner one is chosen for implementation in the low-energy portal film option. Improvement of the image quality was demonstrated with a portal film scale tray and an anthropomorphic phantom.

Galbraith (1989) described a method for portal imaging with low-energy ( $\leqslant 150 \mathrm{keV})$ photons from a radiotherapy accelerator operating in a diagnostic mode. To avoid selfabsorption of the low-energy photons by thick high- $Z$ target, external targets $(\mathrm{Be}, \mathrm{C})$ were employed with the thickness required to stop all the electrons in the target. The targets were positioned at the target slider, outside the bend magnet assembly of an AECL Therac 20 accelerator. If the operating energy was kept low $(\sim 5 \mathrm{MeV})$ and the flattening filter is removed, an estimated $5-18 \%$ of the spectrum lies in the diagnostic region $(\leqslant 150 \mathrm{keV})$. These low-energy photons have the same initial source size and a somewhat similar angular distribution as their high-energy counterparts, and are produced in the same part of the target. As a result, low-energy portal images of a good quality were produced.

In this work, feasibility studies of a new thin target approach, which include calculation analysis of the main physical parameters for the target thickness and composition optimization and its experimental verification, are carried out. The feasibility studies show the great potential capabilities of the new thin-target concept.

\section{Materials and methods}

\subsection{Basic considerations of the thin-target approach}

In a routine radiotherapeutic practice, portal images are taken with the same target port as the radiation treatment itself. For example, in single x-ray mode machines, the portal films are 
taken with the therapeutic port and beam. In dual-energy machines, the portal films are taken with the lower-energy target port and beam, for reasons considered previously. However, in both cases, the physical parameters of the target port used both for irradiation treatment and for portal imaging are determined exclusively on the basis of physical requirements of the therapeutic port (choice of target material, thickness). For this reason the therapeutic target is designed to be more massive and thicker than would be required for a portal image port (lower electron beam energies, lower- $Z$ target materials, lower radiation and thermal loads). As a result, a larger portion of the low-energy part of the total x-ray spectrum is absorbed by the target. Therefore, to solve the problem of excessive self-absorption of low-energy photons by a thick target, we propose to use a thin target with a thickness considerably less than would be required to stop the electron beam in the target (Tsechanski et al 1997b). Moreover, the thickness of such a target, its composition and the electron beam energy all should be determined on the basis of maximum low-energy photon fluence on the upstream side of the target.

\subsection{Monte Carlo simulations}

To determine the optimum parameters of the thin target for portal imaging we have performed Monte Carlo calculations of coupled electron-photon transport using the EGS4 code (Nelson et al 1985, Bielajew et al 1994) with the electron transport enhanced by use of the PRESTA algorithm (Bielajew and Rogers 1986, 1987). The following parameters were used for the simulations: low-energy cut-offs of $0.521 \mathrm{MeV}$ for electrons (EGS4 parameters $\mathrm{AE}$ and ECUT) and $\leqslant 0.050 \mathrm{MeV}$ for photons (AP and PCUT). The Monte Carlo simulations made use of the code EXAMIN to tabulate basic electron and photon data as well the code FLURZ to calculate the fluence of electrons and photons in a cylindrical geometryt. The input parameters included are: electron beam energy, target material (atomic number $Z$ ) and target thickness. The bulk of the calculation involved Monte Carlo simulation of monoenergetic electrons incident normally on slabs of $\mathrm{Cu}, \mathrm{Al}, \mathrm{Be}$ and graphite in the energy range $2-10 \mathrm{MeV}$. The following results were sought:

(i) Transmission photon and electron fluence spectra.

(ii) The ratio of integrated photon fluence, i.e.

$$
\xi=\frac{\int_{0}^{E} \mathrm{~d} E^{\prime} \Psi_{\gamma}\left(E^{\prime}\right)}{\int_{0}^{E_{\max }} \mathrm{d} E^{\prime} \Psi_{\gamma}\left(E^{\prime}\right)}
$$

where $E^{\prime}$ is the photon energy, $\Psi_{\gamma}\left(E^{\prime}\right)$ is the differential photon fluence, $E$ is the upper energy limit for those photons which were considered to contribute to image quality and $E_{\max }$ is the maximum energy of the x-ray spectrum.

The first part of the portal imaging beam Monte Carlo studies involved the investigation of differential photon fluence spectra emitted on the immediate downstream side of a photon target. For this study we employed a $2 \mathrm{~mm} \mathrm{Cu}$ (a common accelerator target material) target and 2, 3, 4, 5, 6 and $10 \mathrm{MeV}$ normally incident monoenergetic electron beams. A compilation of the photon differential fluences as a function of energy for different monoenergetic electron beam energies is presented in figure 1. As can be seen, the photon fluence gets softer with increased electron beam energy. This is because photons are being created ever deeper in the target nearer the exit surface and thus there is less low-energy

$\dagger$ The 'user-codes' EXAMIN and FLURZ are distributed with the unix distribution of EGS4 which is available on the World Wide Web through the central distribution site (http://www.irs.inms.nrc.ca/inms/irs/EGS4/get_egs4.html). 


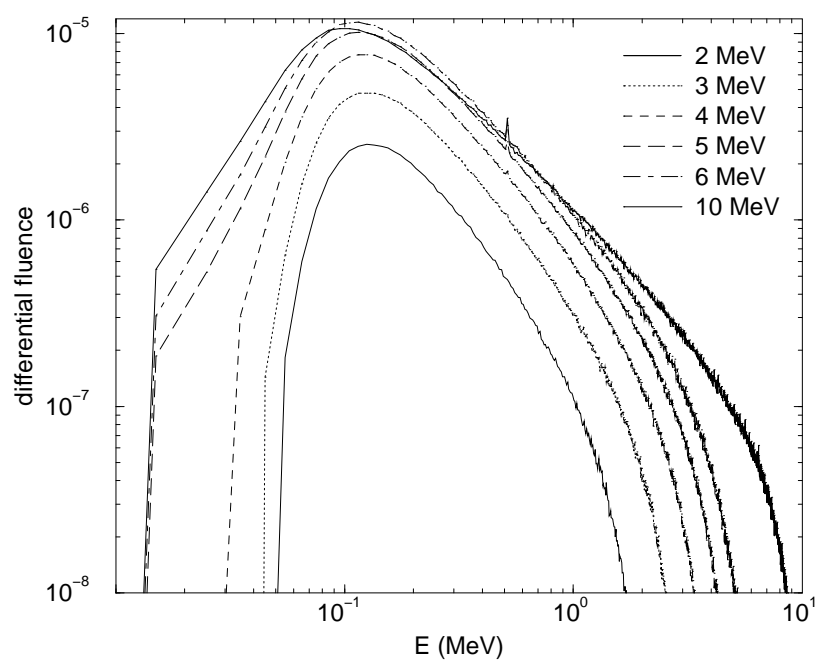

Figure 1. A compilation of the photon differential fluences exiting a $2 \mathrm{~mm}$ slab of $\mathrm{Cu}$ as a function of energy of different monoenergetic electron beam energies.

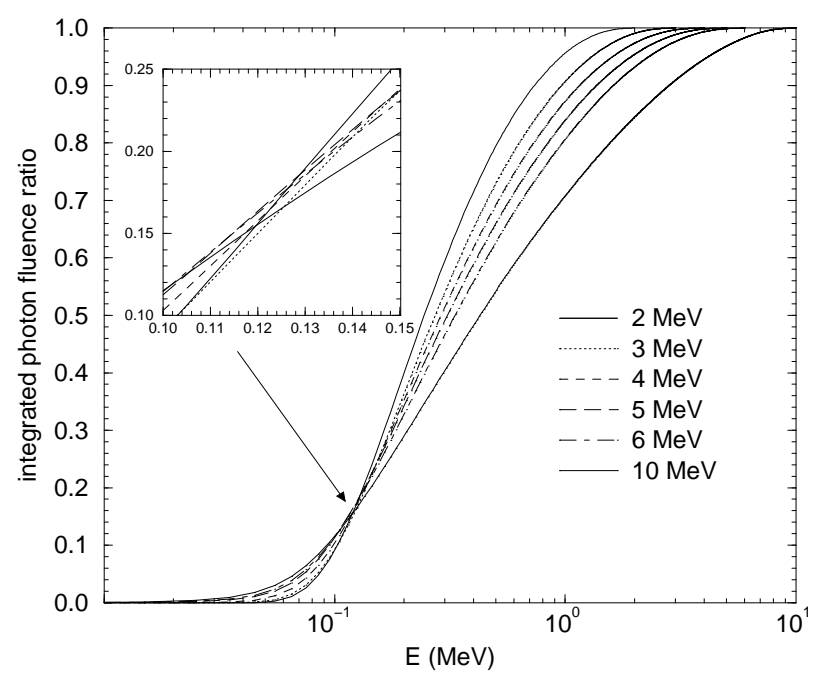

Figure 2. An integrated photon fluence ratio (calculated according to equation (1)) versus photon energy for all photons exiting a $2 \mathrm{~mm}$ slab of $\mathrm{Cu}$.

filtration (self-absorption). The photon fluence increases with energy except for the $10 \mathrm{MeV}$ electron beam energy where much of the electron beam passes through the target and becomes unavailable for bremsstrahlung production.

Even more interesting conclusions can be drawn from figure 2 in which an integrated photon fluence ratio (calculated according to equation (1)) versus photon energy is presented. It is seen that all the curves converge at approximately $120 \mathrm{keV}$. Therefore, if this energy is taken as an upper limit for the low-energy photon range (parameter $E$ in equation (1)), there is no point in lowering the electron beam energy from 10 to $2 \mathrm{MeV}$, at least from the point of view of the gain in the integrated photon fluence ratio. If the upper limit of the 
portal image range is taken to be $150 \mathrm{keV}$, there is close to a $10 \%$ gain in the integrated photon fluence ratio due to lowering the electron beam energy from 10 to $6 \mathrm{MeV}$. On the other hand, further lowering of the electron beam energy from 6 to $3 \mathrm{MeV}$ has virtually no effect on the integrated fluence ratio. It should be pointed out that it does contradict to some extent the accepted conclusion that lowering the electron beam energy obtains a higher integrated photon ratio (Karzmark et al 1993, Galbraith 1989).

To study the effect of target thickness on portal image performance, we concentrated on 4 and $10 \mathrm{MeV}$ monoenergetic electron beams normally incident on $\mathrm{Cu}$ targets of various thicknesses. The $10 \mathrm{MeV}$ value was chosen because it was the only x-ray treatment mode available for our Clinac 18 accelerator. On the other hand, a low-energy unflattened beam for the portal image option was installed by us in the Clinac 18 accelerator and its nominal accelerating potential was determined as being 4 MV (Tsechanski et al 1997a). In this study we confirm that the photon energy hardens with increased target thickness (low-energy filtration) and the electron fluence decreases as primary penetration is controlled. In terms of yield, maximum total photon fluence, the optimum thickness is $1.5 \mathrm{~mm} \mathrm{Cu}$ for $4 \mathrm{MeV}$ electron beam, and $4.0 \mathrm{~mm} \mathrm{Cu}$ for $10 \mathrm{MeV}$ as seen in figures 3 and 4 . The integrated photon fluence ratio versus photon energy for a $4 \mathrm{MeV}$ electron beam is presented in figure 5 for different $\mathrm{Cu}$ target thicknesses. For the $1.5 \mathrm{~mm} \mathrm{Cu}$ target this ratio is considerably higher $\simeq 0.27$ as compared with 0.14 for a $4 \mathrm{~mm}$ thick target for the $150 \mathrm{keV}$ photon energy.

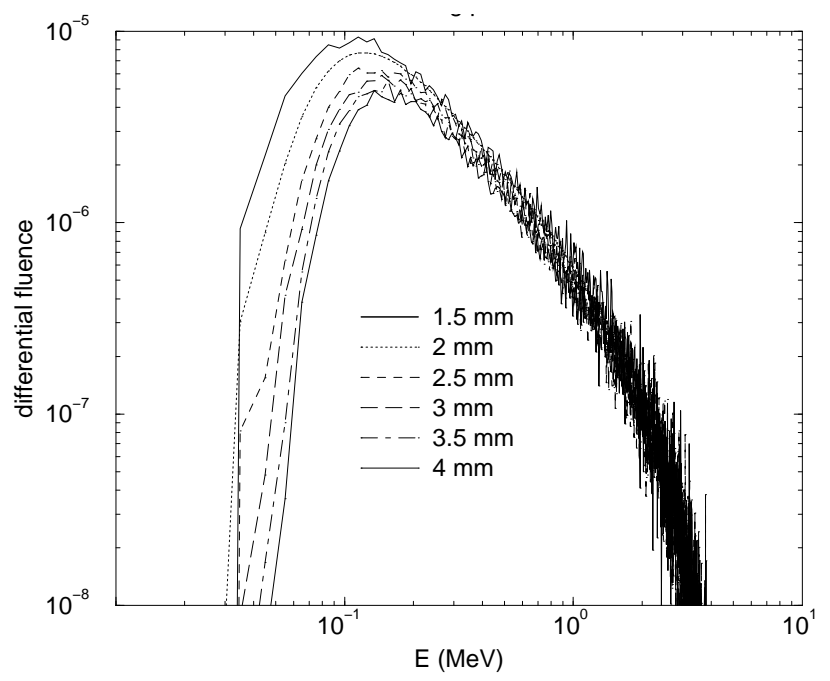

Figure 3. Maximum total photon fluence exiting a $\mathrm{Cu}$ slab from a $4 \mathrm{MeV}$ incident electron beam; the optimum thickness is $1.5 \mathrm{~mm} \mathrm{Cu}$ for a $4 \mathrm{MeV}$ electron beam.

An important part of this study is the investigation of target material $(Z)$, as some publications indicate that lower atomic number materials are preferable for portal imaging (Galbraith 1989, Ostapiak et al 1997). For this purpose, similar Monte Carlo calculations were carried out for the lower- $Z$ materials, Be, C(graphite), $\mathrm{Al}$ and Ti. As a result of these calculations it became evident that the dominant effect in the preservation of low-energy $(\simeq 150 \mathrm{keV})$ photons was not $Z^{2}$ bremsstrahlung process, but the $Z^{4}-Z^{5}$ photoelectric effect self-absorption dependence in the target.

It was decided to continue studying the $4 \mathrm{MeV}$ electron beam exclusively, bearing in mind the results presented in figures 2 and 5. This low energy had already been achieved 


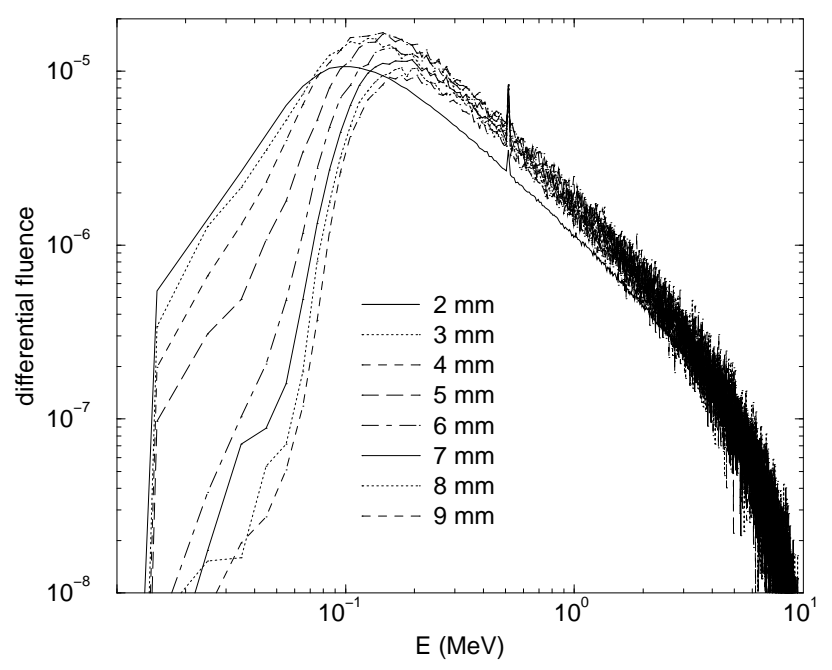

Figure 4. Maximum total photon fluence exiting a $\mathrm{Cu}$ slab from a $10 \mathrm{MeV}$ electron beam; the optimum thickness is $4.0 \mathrm{~mm} \mathrm{Cu}$ for a $10 \mathrm{MeV}$ electron beam.

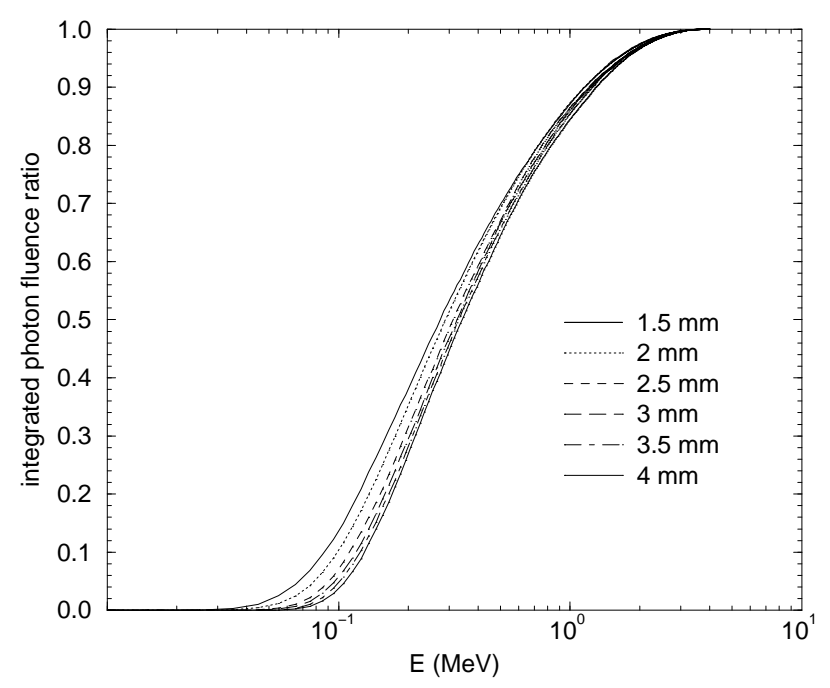

Figure 5. Integral photon fluence ratio versus photon energy for photons exiting a $\mathrm{Cu}$ slab for a $4 \mathrm{MeV}$ electron beam.

in our accelerator and the $4 \mathrm{MV}$ portal images were significantly better than the $10 \mathrm{MV}$ radiographs. We also selected a target thickness of $1.5 \mathrm{~mm} \mathrm{Cu}$, or its equivalent thickness in terms of mass for other materials. In the simulations the region downstream of the target was modelled as a column of air $10 \mathrm{~cm}$ in diameter and approximately $100 \mathrm{~cm}$ in length. The fluence scoring plane was situated in all cases at $100 \mathrm{~cm}$ from the upstream surface of the target. The photon fluence was scored on-axis in a disc $10 \mathrm{~cm}$ in diameter. These simulations were performed with $10 \mathrm{keV}$ cutoffs $(\mathrm{AE}=\mathrm{ECUT}=0.521$ and $\mathrm{AP}=\mathrm{PCUT}=0.01)$. The photon fluences at the $100 \mathrm{~cm}$ plane (unnormalized) are depicted in figure 6 and its lowenergy portion is presented in figure 7 . It should be noted that above $150 \mathrm{keV}$ or so, the 


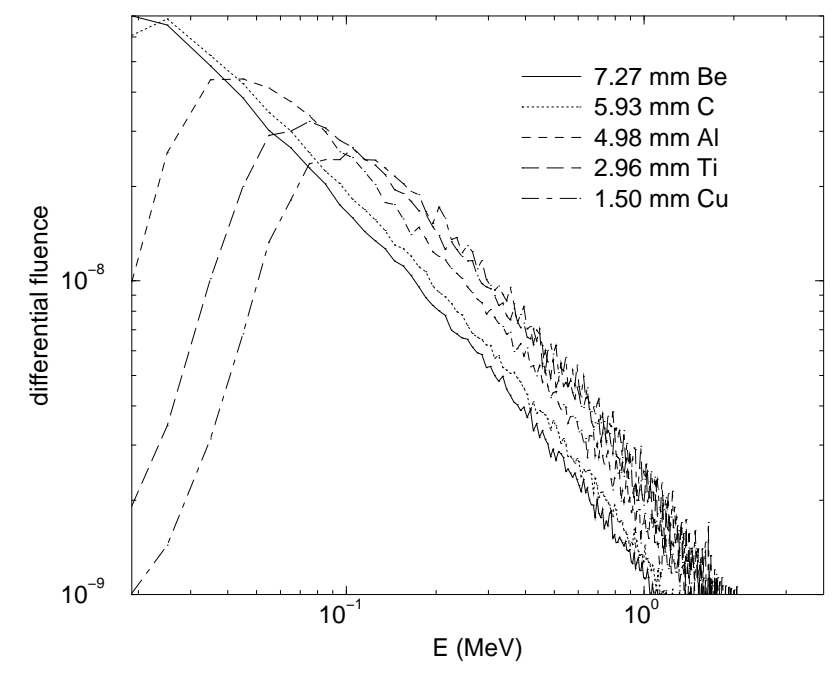

Figure 6. Photon fluences at the $100 \mathrm{~cm}$ plane (unnormalized) for various target materials. Each slab is 'mass-equivalent' to $1.50 \mathrm{~mm}$ of $\mathrm{Cu}$.

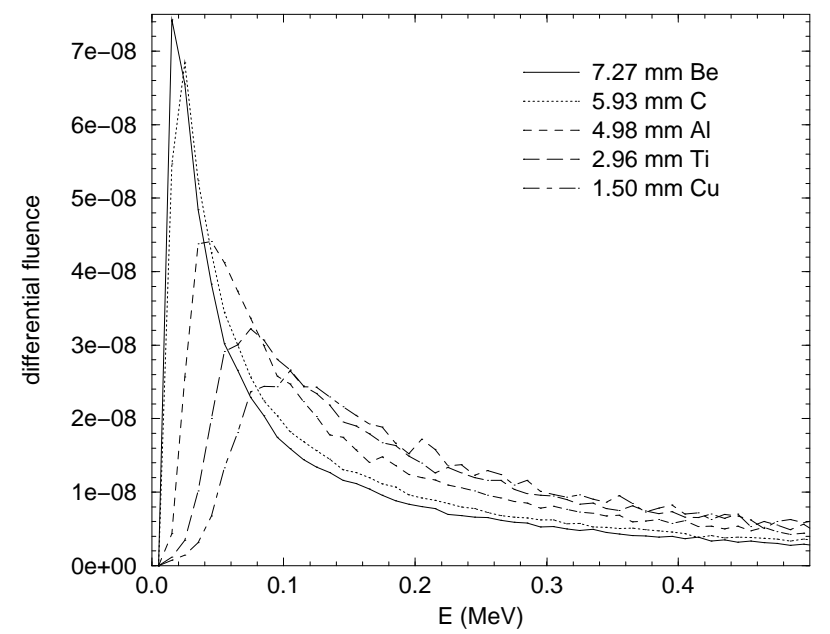

Figure 7. The photon fluence of figure 6 but with the low-energy portion ( $0-500 \mathrm{keV})$ expanded.

spectra are identical in shape. Only a relative normalization distinguished the fluences. The normalized ratio to $\mathrm{Cu}$ is shown in figure 8. Spectra normalization is done at $200 \mathrm{keV}$, giving equal high-energy yields. Finally normalizing to $\mathrm{Al}$ rather than $\mathrm{Cu}$ gives the results depicted in figure 9. Above a photon energy of $150 \mathrm{keV}$ or so, the characteristics of all targets are the same. It is merely the target thickness that determines the relative yield. As seen in figure 9, relative to $\mathrm{Al}$ and below $150 \mathrm{keV}$, Ti absorbs low-energy photons and $\mathrm{Cu}$ even more so. However, down to $50 \mathrm{keV}$ or so, it is not particularly advantageous to use lower- $Z$ materials $(\mathrm{Be}, \mathrm{C})$ as the gain is at most $10 \%$.

It should be pointed out that our preliminary Monte Carlo results which indicate very little target atomic number dependence, are at variance with the suggestions of other authors 


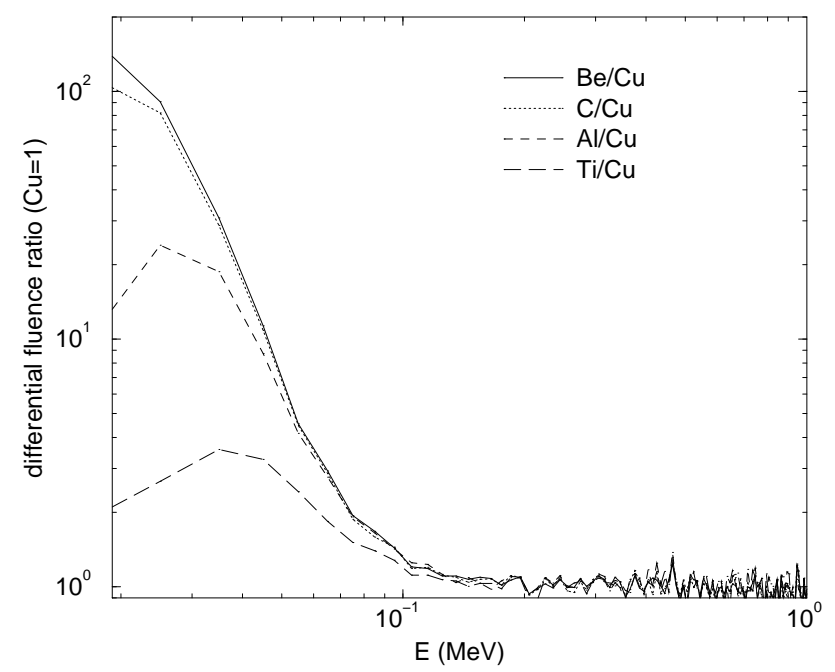

Figure 8. Photon fluence in the low-energy region normalized to the $\mathrm{Cu}$ target photon fluence at $200 \mathrm{keV}$. All targets are 'mass-equivalent' to $1.50 \mathrm{~mm}$ of $\mathrm{Cu}$.

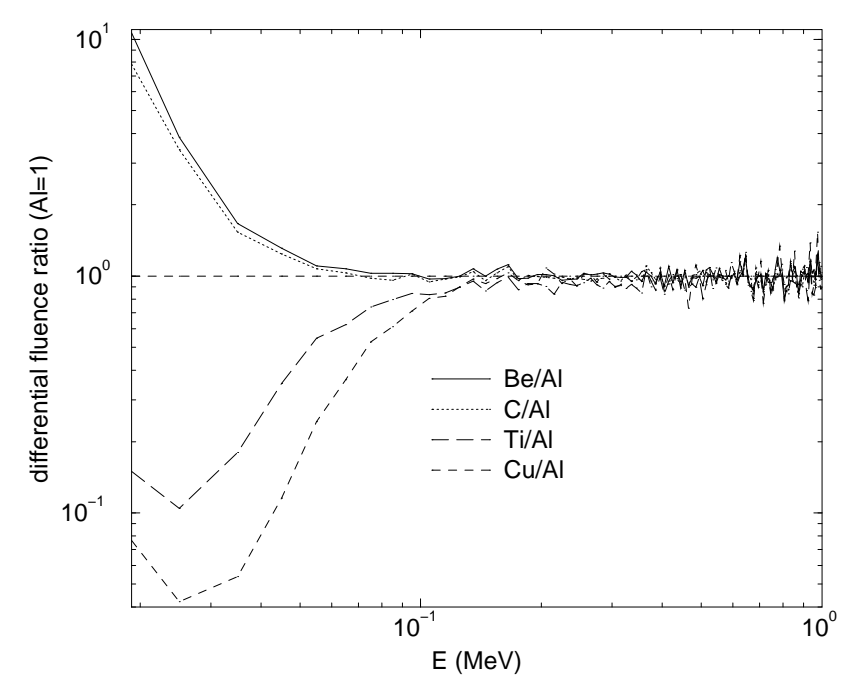

Figure 9. Photon fluence in the low-energy region normalized to the $\mathrm{Al}$ target photon fluence at $200 \mathrm{keV}$. All targets are 'mass-equivalent' to $1.50 \mathrm{~mm}$ of $\mathrm{Cu}$.

(Galbraith 1989, Ostapiak et al 1997) that low atomic number targets, especially Be or graphite, would improve portal image contrast. Compounding the confusion is the result of Faddegon et al (1990), who demonstrated experimentally that the bremsstrahlung yield in the forward direction for thick targets at 10 and $20 \mathrm{MeV}$ is independent of atomic number $Z$. This is the central conclusion of our investigation, that a target thick enough to scatter the electron beam to some extent and to be nearly opaque to photons in the photoelectric regime, but thin enough so that it is nearly transparent to photons in the Compton regime, has its emergent photon spectrum dominated by considerations of the photoelectric absorption cross section. 
Figure 10 represents photon interaction cross sections for the five target materials studied here, $\mathrm{Be}, \mathrm{C}, \mathrm{Al}, \mathrm{Ti}$ and $\mathrm{Cu}$, as well as for air. One sees that the photoelectric cross section starts to dominate at low energies. One should choose a target material that does not absorb photons in the region of interest. For the purposes of portal imaging it appears that aluminium may be a practical choice, especially if a large component of photons in the energy range of approximately $100 \mathrm{keV}$ is desired. If $30-40 \mathrm{keV}$ photons do not contribute to the portal image, it makes no sense to reduce the atomic number of the target to that of $\mathrm{Be}$ or $\mathrm{C}$ since the patient and intervening air attenuate and absorb the extra low-energy photons gained. These extra very low-energy photons (down to $20 \mathrm{keV}$ as seen in figure 7) will have no impact on the portal image quality.

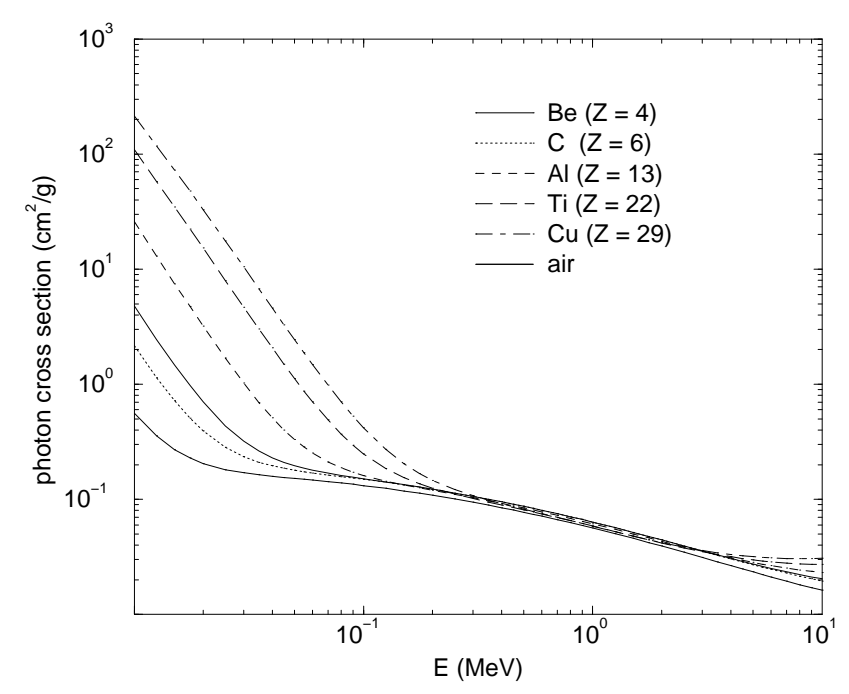

Figure 10. Photon interaction cross sections for the five target materials studied here, Be, C, $\mathrm{Al}, \mathrm{Ti}$ and $\mathrm{Cu}$, as well as for air.

Further simulations confirm our results and are presented in an internal report (Bielajew et al 1996).

\subsection{Experimental set-up}

2.3.1. Target positioning. A low-energy unflattened beam for portal film option was installed and is now routinely used at the Clinac 18 linear accelerator of the Soroka Medical Center. The nominal accelerating potential of the low-energy portal beam option was determined to be $4 \mathrm{MV}$ in accordance with our computations and experimental results (Tsechanski et al 1997a). However, this option cannot be used to check the thin target approach under discussion, since this would require intervention in the existing target assembly located inside the accelerator under vacuum conditions. To avoid a time-consuming and an expensive intervention into the internal (vacuum) structure of the accelerator for changing the target design, it was decided to base the experimental verification of the thin target approach on an external target. The idea of using an external target (outside the vacuum space of the accelerator) is not a new one. In the work of Galbraith (1989), electrons were extracted from an accelerator (Therac 20) through the 
radiotherapy exit window $(0.2 \mathrm{~mm}$ of $\mathrm{Al})$ to strike an external $\mathrm{Be}$ or graphite target inserted into a hole in the target slider. It should be pointed out that the external $\mathrm{Be}$ or graphite targets were chosen thick enough to stop completely the incident electron beam. The same idea underlies the recent work of Ostapiak et al (1997), in which the treatment head of a Siemens MXE Digital Mevatron (Siemens Medical Systems, USA) was modified to allow an external Be target mounted in the collimator to be positioned in the path of the electron beam. In the case of our Clinac 18 linear accelerator, the external thin target of $\mathrm{Cu}$ or Al was installed in the carousel assembly in close proximity to the exit window of the accelerator.

To be useful for radiotherapy the bremsstrahlung beam emerging from the target must be shaped to a beam having definite dimensions and dosimetric characteristics. This is done by the collimation system located in the head of the machine (figure 11). The main components of this subsystem are primary and secondary collimators, flattening filter and scattering foils. One of the important parts of the beam shaping subsystem is the carousel, which is located outside the vacuum environment in close proximity to the exit window of the accelerator in the plane perpendicular to the emerging electron or x-ray beam (figure 11). The carousel (figure 12) is a round plate with a number of symmetrically located openings (ports) on which are mounted a number of electron scattering foils (one foil for one or two electron beam energies) and one or two x-ray flattening filters (one flattening filter for every $\mathrm{x}$-ray mode). The carousel is rotated by means of a stepping motor to bring the appropriate $\mathrm{x}$-ray flattening filter or electron scattering foil into position just under the accelerator exit window and coaxial with it. It should be emphasized that the carousel is located in close proximity to the exit window outside the vacuum space of the accelerator guide. As a result, the carousel can be easily approached to carry out some necessary mechanical adjustments like the removal of a scattering foil and/or installation of an external target.

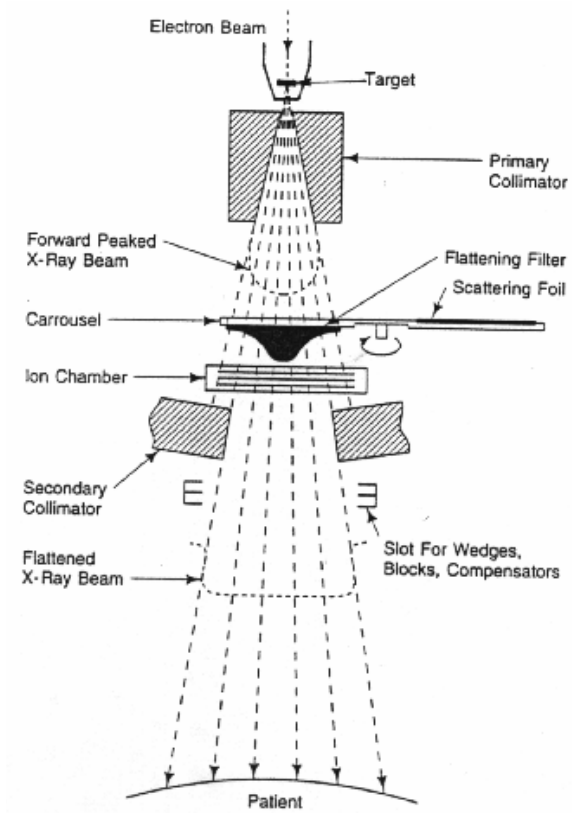

Figure 11. Clinac 18 collimation system (located in the head of the machine). 


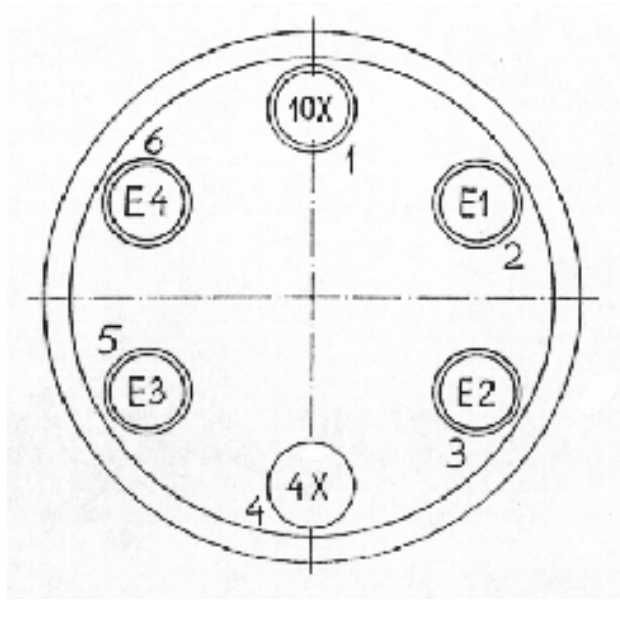

Figure 12. Schematic of the carousel for the Clinac 18 collimation system.

One of the vacant openings of the carousel (such as port 4 in figure 12) routinely used for generation of the $4 \mathrm{MV}$ unflattened portal image beam, was employed by us for experimental verification of the thin target approach for portal imaging applications. For this purpose two sets of experiments were carried out. In the first stage, a $1.5 \mathrm{~mm}$ thick $\mathrm{Cu}$ disc was installed in port 4 (figure 12) of the carousel and used as a thin target. The disc was installed in such a way that the distance between it and the accelerator exit window was as small as possible $(\leqslant 5 \mathrm{~mm})$. In the second stage of the experimental work, the $1.5 \mathrm{~mm}$ $\mathrm{Cu}$ target was replaced by a $5 \mathrm{~mm}$ thick $\mathrm{Al}$ target ( $\mathrm{Cu}$ mass equivalent).

2.3.2. Portal films with copper and aluminium thin targets. To check the performance capabilities of the $1.5 \mathrm{~mm}$ thick external $\mathrm{Cu}$ target we took a number of portal films of a Rando anthropomorphic phantom. The portal films were taken with an unflattened beam generated by the $4 \mathrm{MeV}$ electron beam bombarding the $1.5 \mathrm{~mm}$ thick external $\mathrm{Cu}$ target (figure 13).

To emphasize the low-energy response of the thin target approach, we used a Kodak Min- $\mathrm{R}$ film and a Kodak Min- $\mathrm{R}$ cassette. The Min- $\mathrm{R}$ films are highspeed, orthochromatic, high-contrast, single-emulsion $\mathrm{x}$-ray films specifically designed for mammography applications (Kodak 1996a). Because of its high sensitivity (Kodak 1996b), doses of only $1 \mathrm{cGy}$ are adequate compared with 5 cGy usually required in megavoltage portal imaging (Faermann and Krutman 1992). The light $(\simeq 700 \mathrm{~g}), 24 \mathrm{~cm} \times 30 \mathrm{~cm}$, all-plastic moulded Kodak Min-R cassettes provide easy loading of $\mathrm{x}$-ray films for portal imaging. It should be pointed out that the Kodak X-Omat cassettes, which are generally employed in megavoltage portal imaging, are substantially more cumbersome and heavy, $\simeq 2.7 \mathrm{~kg}$ (Kodak 1988).

A portal image of the head and neck region of a Rando anthropomorphic phantom taken with the $1.5 \mathrm{~mm}$ thick $\mathrm{Cu}$ target, the $4 \mathrm{MeV}$ electron beam and using a Min-R film-screen combination is presented in figure 13. A portal film under the same conditions but with the $5 \mathrm{~mm}$ thick Al target is shown in figure 14 .

To enable a complete comparison between the experimental portal images presented here (figures 13 and 14) and portal films obtained with the usual technique, we present in figures 15 and 16 two additional film images. Figure 15 represents a localization portal film 


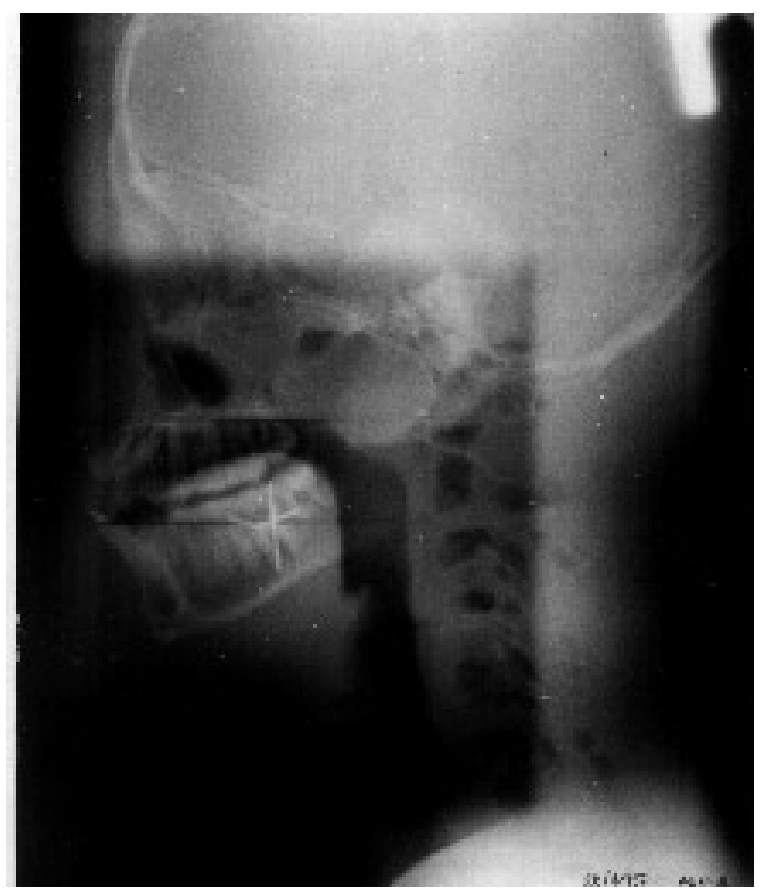

Figure 13. Portal film of the head and neck region of a Rando anthropomorphic phantom, taken with a $4 \mathrm{MeV}$ electron beam bombarding the $1.5 \mathrm{~mm}$ thick (external) target, using a Min- $\mathrm{R}$ film-screen combination.

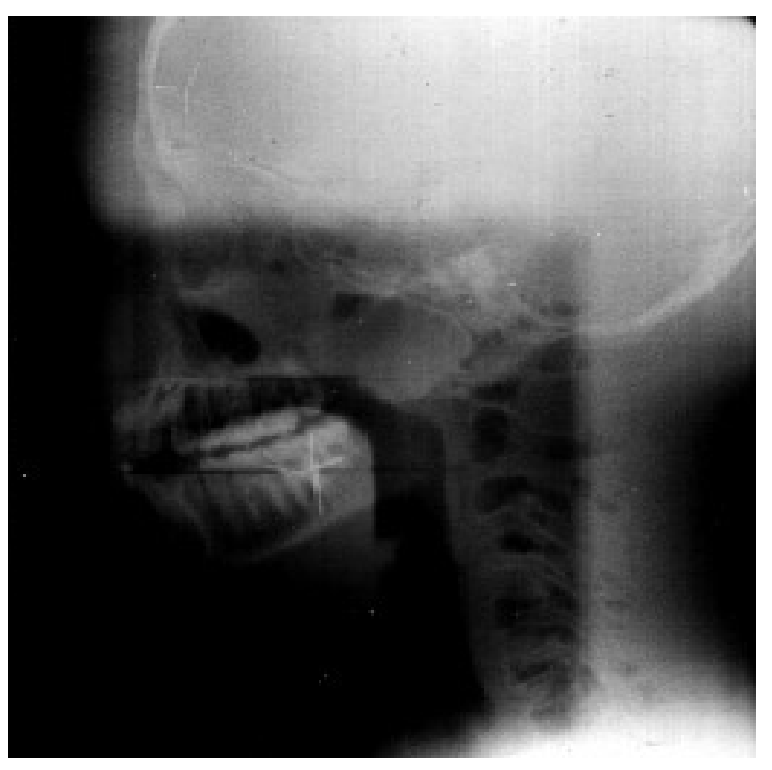

Figure 14. Same as figure 13, but with a $5 \mathrm{~mm}$ thick $\mathrm{Al}$ target.

taken with the $10 \mathrm{MV}$ therapeutic x-ray beam of the Clinac 18 linear accelerator (Soroka Medical Center). The dose to the film was $5 \mathrm{cGy}$. An X-Omat cassette and X-Omat TL 5 therapeutic Kodak film were used (Kodak 1988). It should be pointed out that virtually the 


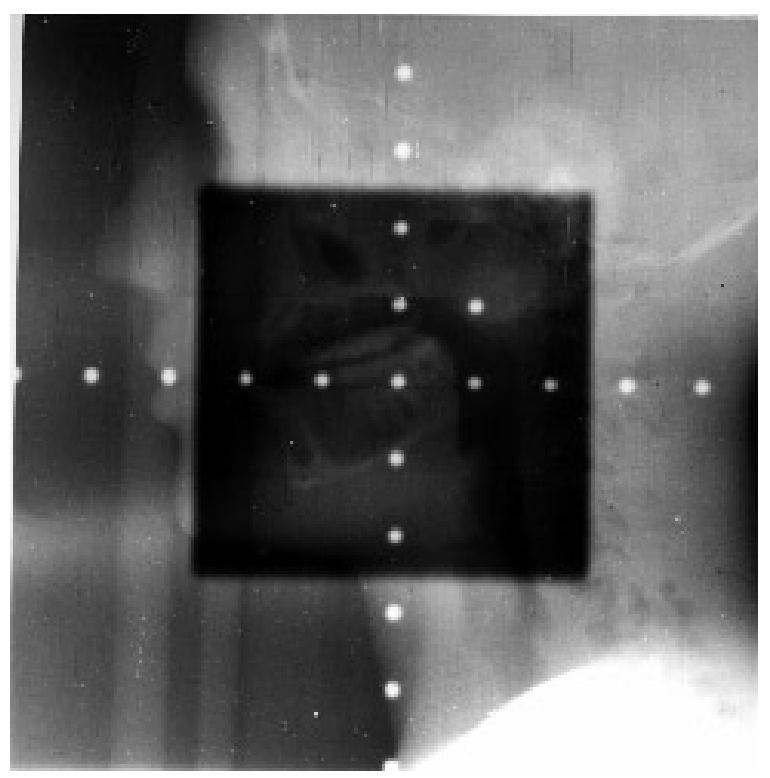

Figure 15. Double-exposure localization film with same set-up as in figure 13, using a $10 \mathrm{MV}$ therapeutic x-ray beam of the Clinac 18 .

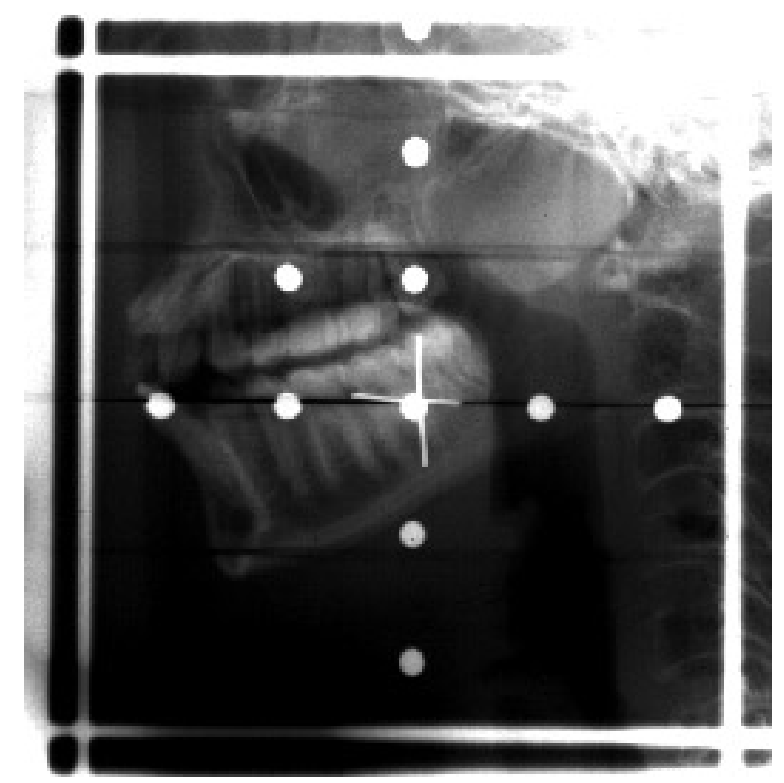

Figure 16. Simulation film of the same set-up like in figure 15 , taken with $150 \mathrm{kV}_{\mathrm{p}}$.

same quality of portal images was obtained with a Clinac $600 \mathrm{C}$ low-energy accelerator with a $6 \mathrm{MV}$ beam.

Finally, figure 16 shows a simulation film of the head and neck region taken with $150 \mathrm{kV}_{\mathrm{p}}$ at a Siemens Mevasim simulator (Siemens AG, Germany) with a diagnostic Fuji film (Fuji Film Corporation, Japan) and Agfa Curix (Agfa-Gevaert Group, Germany) diagnostics cassette. This image serves as a 'best achievable' paradigm. 
All portal films were taken with a double-exposure technique with a field size of $10 \times 10 \mathrm{~cm}^{2}$ and a source-to-skin distance of one $100 \mathrm{~cm}$. The films were developed with a Kodak X-Omat developer at $34{ }^{\circ} \mathrm{C}$ (Faermann and Krutmann 1992).

\section{Results and discussion}

Visual comparison of the thin-target portal films (figures 13 and 14) with a routine portal film (figure 15), clearly demonstrates superior spatial and contrast resolution of the thin target portal images. It is obvious that the enhanced image quality of figures 13 and 14 is undoubtedly advantageous in determination of field placement errors. It should be remarked that the high quality of the Al target portal image (figure 14) with a fine structure clearly seen in teeth and neck regions competes with or even surpasses the quality of the simulator image (figure 16).

An inherent shortcoming of the thin target approach is the presence of a large electron fluence on the downstream side of the target. From figure 3 it can be seen that for a $1.5 \mathrm{~mm}$ $\mathrm{Cu}$ target, the total electron fluence amounts to approximately two-thirds of the total photon fluence. Such an excessive electron fluence can cause a prohibitively high dose on the patient skin and also considerably deteriorate the contrast of portal imaging. To overcome this problem a $0.9 \mathrm{~cm}$ thick plastic tray was inserted into the electron applicator slot of the accessory mount assembly in the accelerator head. The plastic tray is almost transparent to low-energy photons, attenuates the electron fluence considerably and dramatically improves the contrast of the thin target portal images (figures 13 and 14). Additionally, the use of the highly sensitive film-screen combination contributes to minimizing the problem.

Another observation connected with the thin target portal images should be noted. At lower incident photon energies (4 MV), Compton scattering is substantially less forwardpeaked than at higher energies, thus decreasing the probability that a scattered photon will still be in the field at the cassette position. It follows, therefore, that to improve the contrast of low-energy portals one should increase the air gap between the cassette and patient. We have found that the best contrast in the thin target case with the Min- $\mathrm{R}$ film-screen combination is achieved with an air gap of $20 \mathrm{~cm}$, as compared with a $10 \mathrm{~cm}$ gap for a routine portal.

When using the external target employed in this study, the source of x-ray radiation is approximately $10 \mathrm{~cm}$ closer to the phantom than the $\mathrm{x}$-ray source of a routine therapeutic beam. As a result, the portal images obtained are magnified by approximately $10 \%$ as compared with its radiation field counterpart $(\mathrm{SSD}=100 \mathrm{~cm})$. This, together with an increased air gap between the cassette and the phantom, explains why the external target portal images (figures 13 and 14) look magnified when compared with a routine portal image (figure 15).

Finally, a comparison between the thin target portal images (figures 13 and 14) and the routine portal image (figure 15) reveals blurred boundaries of the $10 \times 10 \mathrm{~cm}^{2}$ image in the thin external target case. This feature finds its explanation in the non-coincidence of radiation sources (foci) of the therapeutic and external target modes. As mentioned, in the case of the external target the source is about $10 \mathrm{~cm}$ distant from the original therapeutic target source. As a result, the x-ray beam emanating from the external x-ray target is not tangential to the inner faces of the moving jaws of the secondary collimators, thereby increasing transmission penumbra.

At this point, a number of remarks concerning the thin external target and its portal images seem to be worthwhile. First of all, the problem of cooling down of external targets should be addressed. In this context it should be noted that the radiation, and therefore 
the thermal load on the target in the portal image mode, is approximately two orders of magnitude lower than in a therapeutic mode, especially when the highly sensitive Min- $R$ film-screen combination is used. In addition, since an external target is assembled outside the vacuum enclosure of an accelerator (under ordinary room atmosphere), there is no need for an elaborate water-cooled system for the target, which is usual for routine therapeutic targets. These facts substantially simplify the problem of cooling down of an external target. We did not observe any increase in temperature of the external target as a result of taking portal films. We did not see also any traces of $\mathrm{Al}$ target damage under electron beam bombardment despite its low melting point $\left(660^{\circ} \mathrm{C}\right)$.

\section{Conclusion}

A thin target approach for portal imaging applications is proposed and described. It is shown that low-energy bremsstrahlung photons produced by the accelerator with thin targets $(1.5 \mathrm{~mm} \mathrm{Cu}$ or $5 \mathrm{~mm} \mathrm{Al})$ can be effectively used for producing high-quality portal images. An external target approach can be used for experimental verification of the optimal parameters of the thin target (choice of target material, target thickness and electron beam energy). It is shown that a mammographic film-screen combination can be used successfully in thin target portal imaging with a low-energy unflattened x-ray beam. EGS4 Monte Carlo analysis of coupled electron and photon transport of the thin target approach shows that there is little motivation to use target materials with $Z$ lower than $\mathrm{Al}$ to get high-quality portal images. Further Monte Carlo calculations are desirable, especially with more accurate reproduction of the accelerator head, anthropomorphic phantom and experimental geometry.

In the external target portal imaging case, blurring at the field boundaries is observed due to geometrical distortion because of the non-coincidence between the radiation sources in treatment and portal image modes. To overcome this problem an external target portal image should be digitized and recalculated by means of standard codes. It should be pointed out that such a recalculation would require accurate data about the geometric parameters of the accelerator head assembly, such as target-to-exit-window distance, exit window-tocarousel distance, target thickness and some others. However, these data are proprietary information of the accelerator manufacturer and therefore cannot be easily obtained.

The applicability of the thin-target approach to electronic portal imaging devices (EPID) should be discussed at this point. The different EPID systems were designed based on the use of the exiting therapeutic ports, (thick target and flattening filter in the beam path). Therefore, a low portion of the x-ray spectrum is inevitably absorbed in the beam path. In such a situation there is no need at all to apply a low-energy photon-sensitive detector, like the mammographic film-screen combination used in the present work. Further experimental work is desirable to study the response and compatibility of the existing EPIDs to the lowenergy portion of the x-ray spectrum in connection with the thin-target approach.

A specific radiotherapy treatment to which such a high-quality imaging could be most advantageous is stereotactic radiosurgery in which small, typically $10-30 \mathrm{~mm}$ size tumours or malformations, usually in the head region, are treated by a large radiation dose with high spatial precision, typically within $1 \mathrm{~mm}$.

Another specific treatment which could benefit from this kind of imaging is conformal radiotherapy. For conformal radiotherapy it would be desirable to take daily pretreatment open field images of bony landmarks at orthogonal gantry angles for tumour localization and positioning in three-dimensional space coordinates. It would likewise be very desirable to perform daily pretreatment in-field images at each beam/patient orientation for verification of the field with respect to the patient anatomy. Without bone in the treatment field, in-field 
images alone are inadequate in many situations using megavoltage $\mathrm{x}$-ray (Numan 1985) regardless of dose. The situation will be substantially improved with this new thin-target approach.

\section{Acknowledgment}

This work was partially supported by the Soroka Medical Center.

\section{References}

Amols H I, Reinstein L E and Lagueux B 1986 A quantitative assessment of portal film contrast as a function of beam energy Med. Phys. 13 711-16

Bielajew A F, Faermann S, Krutman Y and Tsechanski A 1996 On the nature of high energy bremsstrahlung beams for portal imaging applications Report (Be'er Sheva, Israel: Institute of Oncology, Soroka Medical Center)

Bielajew A F, Hirayama H, Nelson W R and Rogers D W O 1994 History, overview and recent improvements of EGS4 Report PIRS-0436 (National Research Council of Canada)

Bielajew A F and Rogers D W O 1986 PRESTA: the parameter reduced electron-step transport algorithm for electron Monte Carlo transport Report PIRS-0042 (National Research Council of Canada)

— 1987 PRESTA: The parameter reduced electron-step transport algorithm for electron Monte Carlo transport Nucl. Instrum. Methods B 18 165-81

Evans R D 1955 The Atomic Nucleus (New York: McGraw-Hill)

Faddegon B A, Ross C K and Rogers D W O 1990 Forward directed bremsstrahlung of 10-30 MeV electrons incident on thick targets of $\mathrm{Al}$ and $\mathrm{Pb}$ Med. Phys. 17 773-85

Faermann S and Krutman Y 1992 Generation of portal film charts for 10 MV x-rays Med. Phys. 19 351-3

Galbraith D M 1989 Low energy imaging with high energy bremsstrahlung beams Med. Phys. 16 734-46

Johns H E and Cunningham J R 1983 The Physics of Radiology 4th edn (Springfield, IL: Thomas)

Karzmark C J, Nunan C S and Tanabe E 1993 Medical Linear Accelerators (New York: McGraw-Hill)

Kodak 1988 Kodak Medical Diagnostic Imaging Catalog Kodak Publication no M5-15 (Eastman Kodak Company)

— 1996a Kodak Mammography Systems Catalog (Eastman Kodak Company)

1996b Kodak Min-R M Film Catalog (Eastman Kodak Company)

Nelson W R, Hirayama H and Rogers D W O 1985 The EGS4 Code System Report SLAC-265 (Stanford, CA: Stanford Linear Accelerator Center)

Nunan C S 1995 Radiotherapy apparatus equipped with low dose localizing and portal imaging x-ray source US Patent 5471516

Ostapiak O Z, O'Brien P F and Faddegon B A 1997 Megavoltage imaging with low Z targets: implementation and characterization of an investigational system. Canadian Organization of Medical Physicists Conf. Proc. (Edmonton: Canadian Organization of Medical Physicists Secretariat)

Purdy J A, Gerber R L, Granda C O and Parrino P A 1981 Improved localization films for a high energy linear accelerator Med. Phys. 857

Tsechanski A, Faermann S and Krutman Y 1997a Low energy unflattened beam for portal imaging Phys. Med. 13 $162-5$

Tsechanski A, Bielajew A F, Faermann S and Krutman Y 1997b A target port for high quality portal imaging in linear accelerators: Proc. World Congress on medical physics and biomedical engineering Med. Biol. Eng. Comput. 351064

Varian 1982 Catalog RAD 1933A Varian Report 10/82 (Varian Associates) 